\title{
Morphological requirements in limulid and decapod gills: A case study in deducing the function of lamellipedian exopod lamellae
}

Yutaro Suzuki, Akiyoshi Kondo, and Jan Bergström

Acta Palaeontologica Polonica 53 (2), 2008: 275-283 doi:http://dx.doi.org/10.4202/app.2008.0208

According to one hypothesis, the exopods of extinct lamellipedian arthropods functioned as gills. To evaluate this hypothesis, the growth rates in Limulus polyphemus for total gill surface, average area per single gill lamella and number of gill lamellae are documented. The rates are compared with corresponding rates in decapod crustaceans in order to make deductions on morphological constraints in multi-foliated gills. The growth rates are given as allometric scaling exponents relative to the animal dry-body weight. The comparisons reveal that each allometric exponent is similar among examined species irrespective of differences in gill morphology or animal body plans. The numerical growth of lamellae obviously is much smaller than the growth of the total respiratory surface. To fulfill these trends in multi-foliated gills, the overall profile tends to become conical, with the result that the surface area is a couple of magnitudes larger at the base of the cone than at the tip. This geometrical shape appears to keep the numerical value of the total respiratory area (total lamellar surface) proportional to the cube of the total number of lamellae. The situation is entirely different in animals with lamellipedian exopods. In the latter, lamellae are slender structures carried in a straight row and, as exemplified by Naraoia, their increase in number during the growth is only half that required for the exopod lamellae to have functioned as an arthropod multi-foliated gill cone.

Key words: Arthropoda, Limulidae, Decapoda, allometrty, gills, respiration, functional morphology

Yutaro Suzuki [sysuzuk@ipc.shizuoka.ac.jp] and Akiyoshi Kondo, Institute of Geosciences, Shizuoka University, 836 Ohya, Shizuoka, 422-8529, Japan; Jan Bergström [jan.bergstrom@nrm.se], Department of Palaeozoology, Swedish Museum of Natural History, P.O. Box 5007, SE-104 05 Stockholm, Sweden.

This is an open-access article distributed under the terms of the Creative Commons Attribution License (for details please see creativecommons.org), which permits unrestricted use, distribution, and reproduction in any medium, provided the original author and source are credited. 
FoF Full text $(645.8 \mathrm{kB})$ 